\title{
ALGUNAS REFLEXIONES SOBRE LOS VERBOS MODALES INGLESES: MUNDOS POTENCIALES Y CONTEXTO
}

\author{
Berbeira Gardón, José Luis
}

Universidad de Cádiz, Departamento de Filologia Francesa e Inglesa, Facultad de Filosofia y Letras, Bartolomé Llompart, s/n, 11003-Cádiz, Tfno.956-245009,Fax.956-220444,e-mail: joseluis.berbeira@uca.es

(Recibido Septiembre 1998; aceptado Octubre 1998)

BIBLID [1133-682X (1997-1998): 5-6, 415-434.]

\section{Resumen}

Este trabajo es una réplica a la reserta de nuestro libro Los verbos madales ingleses Estudio semántıcopragmánco, realizada por la Dra Carretero Lapeyre (este volumen). Carretero destaca diversos aspectos problemáticos de nuestro enfoque, proporcionando, para ello, una serie de ejemplos ilustratıvos. En este trabajo nos planteamos rebatir estas criticas una por una, siguiendo el modelo propuesto en el libro reseñado.

Palabras clave: verbos modales, mundos potenciales, potencial y deseable, teoria de la pertinencıa.

\section{Abstract}

This paper is a reply to Dr. Carretero Lapeyre's revıew of our book Los verbos modales ingleses. Estudıo semánico-pragmático (this volume). Carretero draws attention to different problems with our approach, and provides a considerable number of illustrative examples. Nonetheless, I would like to address the issues within Carretero's paper independently and defend the approach followed in our book.

Key words: modal verbs, potential worlds, potential and desirable, relevance theory.

Este articulo es una réplica a la reseña de nuestro libro Los verbos modales ingleses Estudio semántaco-pragmátıco, realizada por Marta Carretero Lapeyre y publicada en este volumen. 


\section{Résumé}

Cet article est une réponse au compte rendu fait par Mme. Carretero de mon libre Los verbos modales ingleses Estudio semántico-pragmático (cf ce volume-c1). Mme. Carretero souligne divers aspects problématıques de notre perspective d'étude et propose à se sujet une série d'exemples illustratifs. Cependant, notre travail essaiera de contredire les critıques effectuéesà partir du modèle suıvı dans le livre en question.

Mots clés: Verbes Modaux, Mondes pontentiels, Pontentiel et Désirable, Théorie de la Pertınence.

\section{Sumario}

0. Introducción. 1. La definición del concepto "mundo potencial" 2 El significado básıco de las formas modales de pasado. 3. La distinción entre los verbos modales y los semimodales. 4 El significado básico de WILL. 5 El sıgnificado básico de MUST. 6. La compiejıdad cognitiva de las denominaciones "potencial" y "potencial y deseable". 7. El proceso interpretatıvo.

\section{Introducción}

En Berbeira Gardón (1996) presentamos un estudio sobre la semántica y la pragmática de los verbos auxiliares modales ingleses CAN, MAY, MUST y SHOULD. Gran parte del libro está dedicada a justificar las ventajas de un enfoque monosemántico. Siguiendo los presupuestos de la teoría de la pertinencia (Sperber y Wilson, 1986, 1995), se defiende que cada uno de estos verbos tiene un significado básico que forma parte de la forma lógica de los enunciados. En el proceso de interpretación, esta forma lógica se enriquece inferencialmente, de acuerdo con el principto de pertinencia, para dar lugar a las diversas interpretaciones pragmáticas, según los factores contextuales. En el capítulo tercero, sugerimos que las oraciones modales están semánticamente especializadas para la representación de mundos potenciales. Un mundo potencial se define como aquel mundo compatible con los conocimientos del hablante acerca del mundo real y que puede ser, o llegar a ser, igualmente real. De este modo, los verbos modales codifican un mismo concepto, el concepto "mundo potencial", y la diferencia semántica entre los mismos estriba en que cada uno de ellos contiene diferente información para la localización de la proposición expresada en dicho tipo de mundo.

En el capítulo dedicado al proceso interpretativo, demostramos que la información semánticamente codificada por el verbo modal contribuye a la construcción de explicaturas de alto nivel y que las diversas interpretaciones de un enunciado modal son producto del enriquecimiento inferencial del concepto "mundo potencial". Basándonos en la distinción que Sperber y Wilson $(1986,1995)$ establecen entre los usos descriptivos e interpretativos del lenguaje, proponemos finalmente una clasificación 
según la cual la forma proposicional de un enunciado modal puede ser empleada para describir diferentes estados de cosas o para interpretar otras proposiciones en virtud de una cierta semejanza entre los pensamientos que dichas proposiciones pueden representar. La expresión de modalidad presenta cuatro posibilidades: la forma proposicional de un enunciado modal puede ser una interpretación de una proposición potencial o potencial y deseable, o una descripción de un estado de cosas igualmente potencial o potencial y deseable. Aquellas interpretaciones en las que interviene la noción de deseo pueden subdividirse a su vez, según el estado de cosas descrito sea deseable desde el punto de vista del hablante o del oyente.

Este enfoque, que más que defender sólo hemos podido presentar, quiere ser muy escrupuloso con las plausibilidad psicológica de los aspectos que intervienen en el proceso de interpretación de enunciados modales, y justifica una división entre la semántica y la pragmática de estos verbos motivada psicológicamente gracias al marco teórico que hemos adoptado. No obstante, ta reseña de la Dra. Carretero (este volumen), cuyos comentarios, tan puntuales $\mathrm{y}$, al mismo tiempo, tan rigurosos, nos gustaría agradecer, nos ha hecho volver a revisar algunos de los aspectos que tratamos en el libro. Comenzaremos por considerar las críticas de la autora a la definición del concepto "mundo potencial", por ser éste uno de los aspectos claves de nuestro modelo.

\section{La definición del concepto "mundo potencial"}

Si bien Carretero muestra su acuerdo con la idea de que los verbos auxiliares modales codifican la representación de un mundo potencial ${ }^{2}$, la autora expresa sus reservas con respecto a la definición que de este tipo de mundo ofrecen Wilson y Sperber (1988: 85) y su utilidad para el estudio de la modalidad. Recordemos que, según los autores (ibidem), los mundos potenciales se definen como "worlds compatible with the individual's assumptions about the actual world, which may therefore be, or become, actual themselves". Según Carretero, esta definición no es válida, pues implica que todas las proposiciones modalizadas pueden ser o llegar a ser verdaderas en el mundo real, cuando existen numerosos enunciados modales contrafactuales (la autora presenta el ejemplo He should have told the truth) en los que la proposicion expresada se refiere a

2 Coincidimos con Carretero en que la codificación de la representación de las oraciones en un mundo potencial no es exclusiva de los verbos modales (de hecho, en ningún momento hemos afirmado lo contrario), sino que es compartida, entre otras expresiones, por adverbios de duda o probabilidad. A este respecto, aún queda por llevar a cabo la tarea de estudiar, de forma sistemática, la relación entre los verbos auxiliares modales y lo que Hoye (1997) denomina los "adverb satellites of the modals".

Pragmalinguistica, 5-6, 1997-1998, 415-434. 
un hecho irreal ${ }^{3}$. Para solucionar este problema y poder encajar este tipo de enunciados en el esquema propuesto en Berbeira Gardón (1996), Carretero propone definir la noción "mundo potencial" simplemente como un mundo distinto del real, constituido por distintos elementos según los tipos de modalidad. Así, en la modalidad dinámica, se trataría de un mundo distinto del real por cuanto incluiria la posibilidad teórica que tiene la proposición de convertirse en verdad en virtud de propiedades intrínsecas de alguna persona o cosa o de circunstancias externas (posibilidad que, dicho sea de paso, existe también en el mundo real); en el caso de la modalidad epistémica por supuestos acerca del mundo real: en la modalidad deóntica, esta posibilidad de que la proposición pueda llegar a ser verdadera estaría determinada por lo que sería deseable para el hablante, el oyente o terceras personas, incluyéndose las nociones de obligeción y permiso: la obligación resulta de la imposición de un deseo, y el permiso supone falta de obligación positiva y negativa. En definitiva, la autora está realmente sugiriendo un análisis en términos no de mundos potenciales sino de mundos posibles. Esta noción, sin embargo, resulta inadecuada a la hora de estudiar los verbos modales, puesto que no refleja la realidad psicológica ${ }^{+}$.

El principio de pertinencia impone, a nuestro juicio, ciertas limitaciones a la hora de construir alternativas válidas al mundo real: ¿cómo adquieren pertinencia las representaciones de realidades alternativas? Para resultar pertinentes, las representaciones de un determinado mundo deben ser descripciones de un mundo no sólo posible sino también potencial, es decir, lo suficientemente similares al mundo real como para poder ser, llegar a ser o haber sido reales en sí mismas. Para ello, un mundo potencial tiene que compartir un número de proposiciones verdaderas con el mundo real. Las representaciones de estados de cosas alternativos pueden alejarse radicalmente de nuestras representaciones mentales del mundo real; sin embargo, todo alejamiento de éste requiere un esfuerzo cognitivo considerable $\mathrm{y}$, por ello, tiene que ser compensado por efectos contextuales adecuados ${ }^{5}$. Estos efectos contextuales reflejan una representación

Es interesante observar que no sólo los enunciados contrafactuales como He should have told the truth se refieren a un hecho ineal. Aquellas proposiciones modalizadas relativas a estados de cosas presentes o futuros también son irreales en el sentıdo de que se refieren a estados de cosas no realizados. Queremos sugerır en este punto que una posible línea de investigación para resolver el problema planteado por Carretero sería la distinción entre dos tipos de potencialidad, a saber, potencialidadreal y potencialidad irreal.

cf. Berbeira Gardón (1998a: 14-15).

De hecho, en un mundo potencial incluiriamos conjuntos de supuestos, ya sean epistémicos o deónticos, junto con un conjunto muy constante de supuestos factuales sobre el mundo real, sin

Pragmalinguistica, 5-6, 1997-1998, 415-434 
distinta de lo que el mundo real podría ser, llegar a ser, o haber sido si se hubieran dado una serie de condiciones. Por otro lado, al invitar al oyente a representarse mentalmente un estado de cosas alternativo, el hablante debe comunicar una representación que el oyente pueda reconstruir a partir de la evidencia disponible. Si éste no encuentra el modo de construir una representación de un mundo posible, preservando al mismo tiempo algunos supuestos fundamentales del mundo real, el enunciado no resultará pertinente, como ocurre en el caso de algunos contrafactuales ${ }^{6}$.

El ejemplo que nos proporciona Carretero (He should have told the truth) efectivamente representa un estado de cosas irreal, es decir, no realizado y, por tanto. no localizado en el mundo real. El hecho de que la proposición expresada "He tell the truth" represente un estado de cosas irreal no significa, sin embargo, que ese estado de cosas no sea potencial. El que una proposición sea potencialmente verdadera no implica necesariamente que lo sea en el presente o en el futuro, sino que esta proposición es compatible con todo lo que el hablante sabe acerca del mundo real y refleja un estado de cosas no realizado, sea cual sea el tiempo en el que se localiza la proposición. Cuando el hablante emite un enunciado contrafactual como He should have told the truth, está presentando la proposición como no realizada y temporalmente la está localizando en el tiempo pasado. ¿Cuál sería la diferencia, entonces, entre esta oración y una declarativa negativa como He didn't tell the truth?. La diferencia estriba en el hecho de que al localizar la proposición expresada en un mundo potencial, los efectos contextuales de la proferencia son distintos. El enunciado modalizado tendría una serie de explicaturas de alto nivel (en las que interviene claramente la actitud de deseo), y, probablemente, daria lugar a implicaturas del tipo If he had told the truth she wouldn't have left him.

Bien es cierto que la definición de mundos potenciales de Wilson y Sperber (1988) no da cabida a la interpretación de aquellas oraciones en las que la proposición expresada se refiere a un hecho pasado, $y$, por tanto, dejaria sin explicar ejemplos como

necesidad de postular la existencia de distintos mundos posibles, como el mundo del razonamiento y la creencia, el mundo de la obligación y el permiso, etc., como se ha hecho en distuntos estudios desde un punto de vista formal y como sugiere Carretero. La inclusión de estos supuestos proporcionaría al Individuo efectos cognitivos que no podría conseguir simplemente con la manipulación de representaciones del mundo real.

- Kratzer (1981) en su análisis de los contrafactuales, señala que at determinar el conjunto de proposiciones pertinentes para asignarles un valor de verdad, sólo deben considerarse aquellas proposiciones que están al alcance del individuo.

Pragmalinguistica, 5-6, 1997-1998, 415-434. 
el presentado por Carretero?. Sin embargo, pensamos que la definición seguiría siendo válida si la reformuláramos del siguiente modo: "worlds compatible with the individual's assumptions about the actual world, which may therefore be, become or have been actual themselves".

\section{El significado básico de las formas modales de pasado}

Las formas modales de pasado reciben un tratamiento secundario en Berbeira Gardón (1996), por tratarse de un punto que, en realidad, dejamos como objeto de estudio para futuras investigaciones. En el tercer capítulo (pág. 119), nos limitamos a sugerir que no es necesario especificar contenidos lingüísticos aparte para las formas de pasado COULD y MIGHT, sino que estas formas, como variantes de pasado de CAN y MAY respectivamente, deben recibir los mismos significados básicos que sus correspondientes formas de presente. Por otro lado, SHOULD se considera como forma de presente y no como forma de pasado de SHALL. Carretero critica este tratamiento de SHOULD, y afirma que sería más adecuado su tratamiento como variante de pasado de SHALL. Hemos de reconocer que la propuesta de Carretero sería más coherente desde un punto de vista puramente gramatical, pues trataría del mismo modo a todas las formas de pasado. Nosotros, sin embargo, queremos sugerir, en este punto, la solución contraria: lo más adecuado, y coherente, seria, quizás, conceder a COULD, MIGHT y WOULD el mismo estatus que a SHOULD, es decir, asignarles significados básicos independientes de los de sus correspondientes formas de presente (contrariamente a lo que se afirmaba en Berbeira Gardón (1996)). Este cambio de rumbo en nuestra concepción de las formas modales de pasado se justifica por dos hechos: (1) COULD, MIGHT, SHOULD y WOULD no se emplean exclusivamente para referirse a estados de cosas pasados, sino también para estados de cosas presentes (Mary could be in her office now) y futuros (You should do what I tell you). (2) La consideración de estos verbos como formas de pasado se debe a criterios exclusivamente morfológicos, pero desde un punto de vista semántico y pragmático ha perdido todo su sentido (como se demuestra en (1)). No obstante, la sistematización de estas formas sigue siendo, por el momento, objeto de futuras investigaciones.

Este hecho puede deberse a que Sperber y Wilson, al definir la noción, se centraran sólo en el modo imperativo.

Pragmalingüistica, 5-6, 1997-1998, 415-434. 


\section{La distinción entre los verbos modales y los semimodales}

En Berbeira Gardón (1996: 116), se afirma que la diferencia entre los verbos modales y los semimodales se explica por el hecho de que los primeros codifican el concepto "mundo potencial", mientras que los semimodales, por el contrario, a pesar de contener en sus significados básicos una información similar a la de los modales correspondientes, no codifican este concepto ${ }^{8}$. En el capitulo de conclusiones, se afirma explícitamente que los semimodales localizan la proposición expresada en el mundo real y forman parte del contenido proposicional del enunciado (págs. 167-168). Según Carretero, con algunos de estos verbos esta distinción sólo es válida para el pasado, puesto que en el presente, en enunciados como Mary has to go to the dentist, la proposición también es potencial. Este hecho nos llevaría a replantearnos las diferencias semánticas entre MUST y HAVE (GOT) TO. Si aceptamos la crítica de Carretero. caben dos soluciones posibles: (a) ambos verbos son sinónimos; (b) MUST y HAVE (GOT) TO codifican el mismo concepto, y se diferencian por el hecho de que la relación entre la proposición expresada y la evidencia contextual es distinta. Ambas soluciones resultan, desde nuestro punto de vista, inadecuadas. La primera, porque, como se demuestra en Berbeira Gardón (1996: 113-115), dos enunciados que tan sólo varíen en el empleo de uno y otro verbo tienen diferentes condiciones veritativas (a este respecto, cf. también Leech, 1971: 77). La segunda, porque implica que HAVE (GOT) TO es un verbo modal y cumple una función de operador proposicional. A nuestro juicio, nuestra definición del concepto "mundo potencial", tal y como ta hemos planteado, explica claramente las diferencias semánticas entre los verbos modales y los semimodales, y por qué éstos últimos no codifican dicho concepto'.

Lo único que tienen en común dos enunciados como (1) y (2):

(1) Mary has to go to the dentist.

(2) Mary should go to the dentist.

En realidad, los verbos semımodales no son objeto de estudio de nuestra investigación y, por ello, no reciben una sección aparte. Nuestra afimación forma parte de un argumento en el que se intenta demostrar que MUST y HAVE (GOT) TO no son sinónimos.

- Según Palmer (1979: 163), "( ) a true modal will have no implication of actuality and so may not be used if actuality is implied, whereas a closely related semi-modal has no such restriction or, at least. that if there is an implication of actuality the semi-modal is preferred (...) For necessity, moreover, HAVE TO must be used rather than MUST, if actuality is implied." (las negritas son nuestras).

Pragmalınguistica, 5-6, 1997-1998, 415-434. 
Berbeira Gardón, J. L. - Algunas reflexiones sobre los verbos modales ingleses...

aparte de la relación de entrañamiento existente entre la proposición expresada y la información contextual, es el hecho de que, en ambos casos, la proposición expresada se refiere a un estado de cosas no realizado. Creemos conveniente notar, sin embargo, que el hecho de que una proposición exprese un estado de cosas no realizado no es suficiente para caracterizarla como potencial (si así fuese, todos los enunciados negativos, por ejemplo, como Susan is not a very good student, serían potenciales, al contener una proposición no realizada: It is not the case that [pusan is a good student]). Para que una proposición se considere potencial debe existir, además, un elemento de compromiso, o expresión de actitud, por parte del hablante: aparte de expresar un estado de cosas no realizado, la proposición debe ser compatible con los conocimientos del hablante. Cuando emite un enunciado modalizado, el hablante, al localizar la proposición en un mundo potencial, comunica explícitamente dicho compromiso. Del significado básico de SHOULD se deduce que en ejemplos como Mary should go to the dentist estamos ante aseveraciones de opinión en las que se expresa un cierto compromiso del hablante, dado que éste afirma que parte de la información contextual entraña la proposición expresada. Esta evidencia, al estar localizada en un mundo potencial, incluye las creencias y deseos personales del hablante. Para que, en el proceso interpretativo, el oyente no emplee este tipo de supuestos, tendríamos que emplear HAVE TO. El empleo de este verbo indica al oyente que las circunstancias que hacen necesaria la actualización del estado de cosas descrito son circunstancias externas que, en la mayoría de los casos, ni siquiera necesitan ser procesadas como supuestos contextuales que interactúen con la proposición para que el enunciado adquiera pertinencia ${ }^{10}$.

\section{El significado básico de WILL}

El significado básico de WILL, al igual que las formas de pasado, se planteaba en Berbeira Gardón (1996), como objeto de futuras investigaciones. No obstante, en un trabajo posterior (Berbeira Gardón, 1997), proponiamos, de modo provisional, un contenido lingiuistico para este verbo ( $p$ is true, and the world type is potential), que también es objeto de crítica por parte de Carretero. Según este significado, WILL comparte con el resto de los modales la noción de potencialidad, pero, en cambio, no relaciona la proposición expresada con ningún conjunto de supuestos (Berbeira Gardón,

De hecho, en el caso de Mary has to go to the dentsst, HAVE TO no opera proposicionalmente sobre la proposicion Mary go to the tentist Este verbo forma también parte del contenido proposicional, cosa que no ocure con MUST y SHOULD.

Pragmalingüistica, 5-6, 1997-1998, 415-434. 
1997: 230)" ${ }^{11}$ Según Carretero, esta caracterización está poco fundamentada. dado que:

es razonable pensar que un hablante, tras escuchar un timbrazo, profiere That will be the postman basándose en datos tales como la forma de llamar o la hora en que el cartero suele llegar.

Al contrario de lo que ocurre con verbos como MUST o MAY, los datos en los que se basa el hablante a la hora de emitir un enunciado con WILL no son pertinentes en el proceso interpretativo. El oyente, al interpretar el ejemplo de Carretero. no emplea supuestos contextuales como la forma de llamar el cartero o la hora en que suele llegar (supuestos que, por otra parte, no necesitan ser mutuamente manifiestos a los interlocutores para que el enunciado resulte pertinente). El oyente no necesita ningún conjunto de supuestos que entrañe la proposición expresada o sea compatıble con ella, sino simplemente supuestos contextuales que interactúen con la forma proposicional (en la que se incluye WILL) para derivar interpretaciones epistémicas o deónticas. En Berbeira Gardón (1996: 118, n.149) se sugiere que aquellos enunciados que contienen WILL y se interpretan deónticamente se emplean siempre en contextos en los que está presente la noción de deseo. Situémos (3):

(3) John will help you write up the letter ${ }^{12}$.

en un contexto en el que es mutuamente manifiesto para los interlocutores que el oyente desea que alguien le ayude a redactar la carta y el hablante sabe que el sujeto oracional se ha ofrecido previamente a hacerlo. En estas circunstancias, este enunciado recibiría una interpretación deóntica. Sin embargo, la verdad de la proposición expresada [p John help you write up the letter] no depende en absoluto del deseo del oyente o de los conocimientos del hablante sobre la buena disposición del sujeto oracional. El hablante, para poder codificar su mensaje, ha empleado ambos supuestos contextuales, pero la actualización del estado de cosas que se describe en la proposición va más allá del control y de los conocimientos del hablante.

Lo mismo ocurre en el caso de las interpretaciones epistémicas. Considérese el ejemplo presentado por Carretero:

Esta visión la comparten Sweetser (1990' 54-55), Ehrman (1966) y Groefsema (1995). Ésta última parafrasea la propuesta de Ehrman en los siguientes términos: " don't worry about the evıdence. concentrate on the proposition that will modıfies'" (Groefsema, 1995: 63).

potential.

La forma lógica seria: $\left[_{p}\right.$ John help you write up the letter $]$ is true, and the world type is

Pragmalinguistica, 5-6, 1997-1998, 415-434. 
Berbeira Gardón, J. L. - Algunas reflexiones sobre los verbos modales ingleses...

(4) That will be the postman ${ }^{13}$.

Este ejemplo se interpreta epistémicamente porque no está presente la noción de deseo. La evidencia en la que se basa el hablante para emitir (4) se limita, por tanto, a sus propias creencias personales, de ahí que Carretero afirme correctamente que al emitir un enunciado con WILL, como (4), el hablante se base en información contextual como la forma de llamar o la hora de llegar el cartero.

En el proceso interpretativo, sin embargo, el oyente no emplea ninguno de estos supuestos en los que se basa el hablante (de hecho, éstos ni siquiera necesitan ser mutuamente manifiestos a los interlocutores ${ }^{i 4}$ ). El oyente, al interpretar (4), se limita a procesar la proposición expresada como (potencialmente) verdadera, confiando en el hablante, que presenta dicha proposición como compatible con lo que él sabe acerca del mundo real.

En definitiva, al emitir un enunciado que contenga WILL, el hablante garantiza la verdad de la proposición sin necesidad de ninguna evidencia que la haga verdadera. El oyente, por su parte, no necesita en el proceso interpretativo emplear los supuestos contextuales que el hablante ha manejado en el proceso de codificación.

\section{El significado básico de MUST}

Según Carretero, el signiticado básico que proponemos para MUST "parece demasiado fuerte, al indicar que toda la evidencia de que el hablante dispone vincula la proposición expresada". Según la autora, MUST "evoca la evidencia favorable, lo cual no quiere decir que el hablante carece de evidencia desfavorable". Además, el significado que asignamos a MUST, según Carretero, excluye la posibilidad de otras expresiones modales más fuertes, como aquéllas en las que este verbo admite la continuación con cláusulas como but I don't know (Sue must have lied, but I don't know) o con preguntas arrastre del tipo Sue must have lied, hasn't she?.

No somos de la opinión de que en estos ejemplos el hablante cuente con evidencia desfavorable. Bien es verdad que éste plantea su posible existencia, pero dicha evidencia le es desconocida. En el ejemplo To judge by his look, Peter must be having a good time, although his cheerfulness may be only apparent, la cláusula en la que se

Este enunciado tendria la sigulente forma lógica: ${ }_{p}$ That be the postman] is true, and the world type is potential.

A la sumo, el oyente podría emitir una pregunta del tipo How do you know?

Pragmalinguistica, 5-6, 1997-1998, 415-434 
presenta la evidencia desfavorable está modalizada epistémicamente, lo cual indica la duda del hablante sobre la verdad de la proposición en la que se describe dicha evidencia. En los casos de Sue must hove lied, but I don't know y Sue must have lied, hasn't she?, el hablante implica la posible existencia de evidencia desfavorable. No obstante, dado que esa evidencia le es desconocida, puede seguir manteniendo que todo lo que él sabe sobre el mundo real entraña la proposición. Como se defiende en Berbeira Gardón (1997), estos casos se explican sin dificultad alguna mediante las nociones de mundo potencial y pertinencia dependiente.

\section{La complejidad cognitiva de las denominaciones "potencial" y "potencial y deseable"}

En otro punto, Carretero cuestiona si la denominación "potencial y deseable" conlleva mayor complejidad, desde un punto de vista cognitivo, que la denominación "potencial". En Carretero (1995) se plantea la cuestión de la mayor o menor complejidad de las acepciones epistémicas en comparación a las acepciones dinámica y deóntica. La autora (1995: 41) llega a la conclusión de que las acepciones epistémicas son cognitivamente más complejas que las otras dos, utilizando, entre otros argumentos, la distinción expuesta en Berbeira Gardón (1993) entre los sentidos epistémicos como usos interpretativos del lenguaje y los sentidos radicales (es decir, dinámicos y deónticos) como usos descriptivos (Carretero, 1995: 153, n.14). Lo que Carretero plantea en esta ocasión, sin embargo, es la mayor o menor complejidad cognitiva de la modalidad dinámica en comparación a la modalidad deóntica.

En Berbeira Gardón (1996) se defiende que la noción de "potencialidad" está semánticamente codificada en las oraciones modalizadas, mientras que la noción de deseo se infiere pragmáticamente empleando supuestos contextuales que establecen si el estado de cosas descrito es deseable para alguno de los interlocutores o para una tercera persona o institución. Esto significa que mientras que la noción de deseo sólo está presente en algunos contextos (en concreto, en aquellos en los que el enunciado se interpreta deónticamente), la caracterización del estado de cosas que se describe en la proposición como potencial es inevitable, puesto que esta noción está semánticamente codificada. Por ello, estamos en desacuerdo con Carretero cuando interpreta que en el ejemplo (4.22)(pág. 152) (que aquí repetimos como (5)):

(5) When you are in the neighbourhood, you must come and visit us.

descartamos la descripción potencial antes de inferir la descripción potencial y deseable. El proceso interpretativo de (5) es el siguiente. En primer lugar, el oyente recupera la

Pragmalinguistica, 5-6, 1997-1998, 415-434 
forma lógica (5'):

(5) [ $_{p}$ When you are in the neighbourhood, you come and visit us] is entailed by the set of all propositions which have a bearing on $p$, and the world type is potential.

Una vez enriquecida inferencialmente esta forma lógica, (5') daría lugar a la forma proposicional (5'):

$\left(5^{\circ}\right) \quad[p$ When hearer is in the neighbourhood 1 , hearer come2 and visit speaker and speaker's housemate3] is entailed by the set of all propositions which have a bearing on $p$, and the world type is potential.

en la que el estado cosas se describe como potencial. A partir de esta forma proposicional, y en función de la información contextual (según la cual el estado de cosas descrito hace referencia a un tiempo futuro y su actualización está más allá del control del hablante), el oyente inferirá que, dado que el hablante no puede disponer de ningún supuesto contextual que entrañe la proposición expresada, dicho estado de cosas no sólo es potencial, sino también deseable, por lo que construirá la explicatura de alto nivel $(5 ")$ :

$\left(5^{\prime \prime}\right)$ Speaker desires that $\left[_{p}\right.$ When hearer be in the neighbourhood, hearer come and visit speaker and speaker's housemate].

La combinación de la explicatura o forma proposicional ( $5^{\prime}$ '), en la que el estado de cosas descrito se caracteriza como potencial (y fuera del control del hablante, al estar bajo el control del oyente) y la explicatura de alto nivel (5" ), por la que dicho estado de cosas se describe como deseable para el hablante, da lugar a la interpretación global del enunciado $\left(5^{\prime \prime}\right.$ "):

(5"') Speaker invites the hearer to come and visit speaker and speaker's housemate when he is in the neighbourhood.

Según este procedimiento de comprensión, la descripción "potencial" no se descarta previamente antes de inferir la descripción "potencial y deseable", como afirma Carretero. En la modalidad deóntica, ambas descripciones se recuperan por separado (de hecho, como afirman Wilson y Sperber (1993), un enunciado tiene más de una explicatura). Por ello, la cuestión de una mayor o menor complejidad cognitiva de la modalidad dinámica en comparación a la deóntica nos parece, por el momento, 
irrelevante tanto desde un punto de vista pragmático como cognitivo.

Con respecto a los problemas de adquisición planteados por Carretero, queremos expresar nuestro acuerdo con la autora cuando afirma que "no existen indicios claros de que la modalidad dinámica se adquiera antes que la deóntica", si bien, la adquisición de los distintos tipos de modalidad no han sido, hasta ahora, objeto de investigación por nuestra parte ${ }^{15}$.

\section{El proceso interpretativo}

Uno de los aspectos que Carretero pone en tela de juicio con respecto a la cuádruple clasificación que presentamos en el último capítulo es el tratamiento que reciben las interrogativas modales. En nuestro trabajo, éstas se caracterizan por ser casos de usos interpretativos en los que se interpretan proposiciones potenciales y deseables. Según Wilson y Sperber (1988), las oraciones interrogativas son doblemente interpretativas, por cuanto representan interpretativamente un pensamiento del hablante que, a su vez, es una representación interpretativa de un pensamiento deseable. Si a la caracterización de las oraciones interrogativas de Wilson y Sperber añadimos nuestra caracterización de las oraciones modalizadas como aquéllas en las que se codifica la noción "mundo potencial", podemos concluir que los enunciados interrogativos modales son aquéllos que se emplean interpretativamente para representar un pensamiento potencial y deseable. Carretero arguye, correctamente, que el carácter interpretativo de estos enunciados se debe al modo oracional y no al verbo modal. De hecho, en nuestro trabajo no se afirma en ningún momento que un verbo modal pueda ser empleado descriptiva o interpretativamente, puesto que la posibilidad de ser empleados de uno u otro modo es una propiedad de la forma proposicional de los enunciados y no de los verbos modales o de ninguna otra categoría gramatical ${ }^{16}$. El verbo modal no es responsable de que el enunciado en el que se integra se interprete descriptiva o interpretativamente. El argumento de Carretero, según el cual la conversión de una oración modalizada declarativa en interrogativa no cambia las explicaturas debidas a los

Is Un trabajo interesante en este sentido es Papafragou (1997). En Berbeıra Gardón (1998b) se defiende la idea de que, desde un punto de vista diacrónico, el desartollo de los signuficados dinámico y deóntico se produce en paralelo.

‘ Lo mismo cabría decir en el caso de la metáfora. Cuando emutimos un enunciado como John is a bulldozer, no estamos ante un uso interpretativo del sustantivo BULLDOZER, sino ante un enunciado empleado interpretativamente.

Pragmalinguistica, 5-6, 1997-1998, 415-434. 
modales, sino solamente las debidas al modo oracional, corrobora nuestra tesis ${ }^{17}$.

Nuestro tratamiento de la modalidad deóntica también es objeto de critica por parte de Carretero. Según la autora, el carácter desiderativo de un estado de cosas desde el punto de vista del hablante o del oyente también puede estar presente en enunciados modales epistémicos como You must be earning a good salary now, lo cual puede dar lugar a confusión. Carretero propone añadir otro rasgo a la caracterización de la modalidad deóntica: el control del hablante, el oyente o una tercera persona para llevar o haber llevado a cabo la acción expresada en la proposición. Este rasgo explicaría, según la autora, por qué este ejemplo se emplea epistémicamente: MUST expresa modalidad epistémica porque el salario del oyente está fuera del control del hablante. Esta propuesta nos parece innecesaria y, al mismo tiempo, inadecuada, pues implica la existencia de enunciados epistémicos que se caracterizan como tales porque expresan un estado de cosas deseable para el hablante, aunque fuera de su control. En You must be earning a good salary now (o en ejemplos como He must be very shy) no se infiere la noción de deseo y el control del hablante, el oyente o una tercera persona es irrelevante. Esta noción (empleada por autores como Klinge (1993) en el análisis de los verbos modales) no es objeto, en nuestro trabajo, de un tratamiento pormenorizado, al no tratarse más que de un tipo de supuesto contextual que puede afectar a las explicaturas de alto nivel de un enunciado modalizado (en nuestro trabajo, sólo hacemos referencia a dicha noción cuando el proceso interpretativo del ejemplo lo requiere por motivos de claridad ${ }^{18}$, si bien intuimos que, efectivamente, es común a todas las descripciones de estados de cosas potenciales y deseables).

Para demostrar la transparencia de nuestra descripción "potencial y deseable", emplearemos el siguiente ejemplo:

(6) You must be very careful.

\footnotetext{
Carretero vuelve a hablar de usos descriptivos e interpretativos de los verbos modales cuando afirma: "el uso epistémico de un modal en una interrogativa supondría la superposición de dos usos interpretativos, lo cual resulta extraño (Must John be tall ${ }^{\circ}$ ). Es decir: los modales en oraciones interrogativas tıenen casi siempre un uso descriptivo, aplicado a un estado de cosas potencial (...), o bıen potencial y deseable (2a), que cae dentro del ámbito de un uso interpretativo señalado por el modo oracional." Por otra parte, Carretero cita a Palmer (1990: 62-63), según el cual, las interrogativas modales epistémicas son gramaticales, aunque poco frecuentes. Desde nuestro punto de vista, ninguno de los ejemplos presentados por Palmer son casos de modalidad epistémica, aunque no nos detendremos en este punto.
}

Cf. ejemplos (4 1), (4.22) y (4.11) (pág. 156). 
Este ejemplo puede recibir tanto una interpretación deóntica como epistémica. Según se deduce de la propuesta de Carretero, se interpretaría deónticamente en aquellos contextos en los que el estado de cosas es potencial y deseable $\mathrm{y}$, además, su actualización está bajo el control de alguno de los interlocutores, en este caso del oyente. Si la actualización de dicho estado de cosas es potencial y deseable pero no está bajo el control del oyente, el enunciado recibirá una interpretación epistémica. Según nuestro análisis, ambas interpretaciones tendrían una forma proposicional similar. En la interpretación deóntica, la forma proposicional seria (6'):

(6') [ ${ }_{p}$ Hearer be very careful when hearer go to Thailand] is entailed by the set of all propositions which have a bearing on $p$, and the world type is potential.

Esta forma proposicional tendria que interactuar con la información contextual para dar lugar a las explicaturas de alto nivel. Supongamos que el oyente informa al hablante de que va a realizar un viaje a Tailandia y éste último emite (6). En el proceso de interpretación, el oyente tendrá que recuperar todo el conjunto de proposiciones que entrañan la proposición expresada. Este conjunto podría estar formado por supuestos contextuales como los siguientes: (a) En Tailandia hay un elevado índice de delicuencia y de estafas a turistas; (b) La epidemia de SIDA está creciendo, sobre todo debido a la prostitución; (c) El tráfico es caótico, fundamentalmente en Bangkok; (d) Es peligroso alquilar un coche por tu cuenta, etc. Dado que el oyente no desea ser objeto de un atraco ni una estafa, ni contraer el SIDA, ni encontrarse con problemas en caso de alquilar un coche, inferirá que el hablante le está comunicando que el estado de cosas que se describe en la proposición es deseable para él, y recuperará la explicatura de alto nivel $\left(6^{\prime \prime}\right)$ :

(6') It is desirable to the hearer that [p Hearer be very careful].

Comunicar al oyente que un estado de cosas es potencial ( $y$, en este caso, su actualización está bajo su controi) y deseable desde su propio punto de vista (para evitar estados de cosas no deseables como los que se describen en el conjunto de supuestos contextuales empleados en el proceso interpretativo) es aconsejarle la actualización de dicho estado de cosas.

Contrariamente a lo que sugiere Carretero, la noción de deseo no juega ningún papel en el proceso interpretativo cuando (6) recibe una interpretación epistémica. En este caso, la forma lógica es la misma que en la interpretación anterior, pero ha de ser enriquecida inferencialmente para llegar a la forma proposicional del enunciado (6' '):

Pragmalinguistica, 5-6, 1997-1998, 415-434 
(6"') [ $_{p}$ Hearer be very careful] is entailed by the set of all propositions which have a bearing on $p$, and the world type is potential. ${ }^{19}$

Una vez recuperada esta forma proposicional, el oyente tendrá que considerar cuál es el conjunto de proposiciones que entraña la proposición expresada. Este conjunto se reduce a las creencias personales del hablante (en este caso acerca del oyente), a saber: el oyente es muy esmerado, escrupuloso, metódico, meticuloso, etcétera, y no existe ningún supuesto contextual que haga referencia al hecho de que la proposición expresada sea deseable para alguno de los interlocutores ${ }^{20}$. De ahi que el enunciado recrba una interpretación epistémica.

Por último. en su revisión de nuestro tratamiento de la modalidad deóntica, Carretero se refiere a los distintos subtipos de interpretación, tales como la obligación, el permiso, el mandato, el consejo y la advertencia. En Berbeira Gardón (1996), el hecho de que un enunciado modalizado se interprete como un mandato o como una concesión de permiso se explica según el estado de cosas descrito sea deseable para el hablante o para el oyente, y la distinción entre estas interpretaciones pertenece al ámbito de las explicaturas de alto nivel. Según Carretero, esta explicación es problemática, puesto que la pertenencia a un subtipo u otro no implica cambios en la forma proposicional. Según la autora:

You can help me file these documents podría interpretarse como un mandato si lo comunica su jefe a su empleado, o como una concesión de permiso si lo comunica un padre a un hijo ansioso por ver sus papeles. En los dos casos, can describe un estado de cosas potencial y deseable, de modo que se completa la forma proposicional; las interpretaciones de mandato o permiso son inferencias cancelables que el hablante elabora teniendo en cuenta su relación con el oyente, perteneciendo, por tanto, al ámbito de las implicaturas: si el padre continúa el mensaje con this room needs tidying, el permiso se convertiria en mandato.

Es unteresante notar que, en este caso, la forma proposicional, producto del enriquecimiento inferencial de la forma lógıca, no requiere la asignación de referencia temporal.

Del mismo modo, en el ejemplo de Carretero, You must be earning a good salary now, el conjunto de proposiciones que entrañan la proposición "You be earning a good salary now" se reduce a las creencias del hablante. En este caso, dicho conjunto estaria formado por suspuestos contextuales como los siguientes. (a) el oyente gasta mucho dinero últimamente; (b) el oyente se ha comprado una casa nueva, etc. Este ejemplo se considera como una interpretación de una proposición potencral precisamente porque no está presente la nocıón de deseo.

Pragmalınguistica, 5-6, 1997-1998, 415-434 
Tanto si se interpreta como un mandato o como un permiso, la forma lógica del ejemplo que nos ofrece Carretero es la misma, a saber:

(7) [o You can help me file these documents] is compatible with the set of all propositions which have a bearing on $p$, and the world type is potential.

Los cambios en la forma proposicional no los implica la pertenencia a un subtipo de interpretación u otro, sino el contexto en el que se emite este ejemplo. En el primer contexto, la forma lógica ( $\left.7^{\prime}\right)$ se enriquece inferencialmente para dar lugar a la forma proposicional (7'):

(7') ${ }_{p}$ Employee help boss file documents1] is compatible with the set of all propositions which have a bearing on $p$, and the world type is potential.

La forma proposicional resultante en el segundo contexto, sería la siguiente:

(7"') [ Son help father file documents1] is compatible with the set of all propositions which have a bearing on $p$, and the world type is potential.

Esta forma proposicional sería común a las interpretaciones de mandato y permiso. Carretero apunta que ambas nociones son inferencias cancelables que dependen de la relación entre hablante y oyente, $\mathrm{y}$, por ello, pertenecen al ámbito de las implicaturas. Esto se demuestra, según la autora, porque si el padre continúa el mensaje con this room needs tidying, el permiso se convertiria en un mandato. Esta afirmación, que nos recuerda a la pragmática griceana, nos parece poco fundamentada. Para que este ejemplo se interprete como una concesión de permiso, debe darse necesariamente la condición de que el hablante se represente el supuesto contextual implicado My son desires to help me file these documents. Si, en este contexto, el hablante continúa el mensaje con this room needs tidying, el enunciado no sólo no resultará pertinente, sino más bien absurdo. Sólo en el caso de que el estado de cosas sea deseable para el hablante, se interpretará el ejemplo como un mandato y tendrá sentido continuar el enunciado con this room needs tidying ${ }^{21}$. La propiedad de la cancelabilidad de las implicaturas, a la que hace referencia Carretero, no juega ningún papel en el

Il Según Deırdre Wilson, (comunıcación personal), la noción de deseo, en las interpretaciones de permiso, se inferirá como un supuesto contextual implicado, mientras que en la interpretación de mandato se recuperará como una explicatura de alto nivel. 
funcionamiento real del proceso comunicativo.

Con estas reflexiones, intentamos responder a las puntualizaciones de la Dra. Carretero. Hemos realizado estas nuevas observaciones a partir de las propuestas teóricas ya presentadas en trabajos anteriores (Berbeira Gardón 1993, 1996, 1997) para aclarar problemas en ellas tratados pero que, en su momento, y por motivos ya comentados en cada caso, no fueron resueltos de manera explícita. No obstante, el hecho de que todas estas reflexiones puedan hallar su explicación dentro del mismo modelo, demuestra el carácter generalizador que para el mismo nos proponíamos en dichos trabajos. $Y$, obviamente, los comentarios y sugerencias de otros autores siempre nos resultarán atractivos, sobre todo para seguir profundizando en un tema tan específico y $\tan$ continuamente renovado como la modalidad. 
Berbeira Gardón, J. L - Algunas reflexiones sobre los verbos modales ingleses...

\section{Referencias}

BERBEIRA GARDÓN, J. L. (1993), La pragmática de los verbos modales ingleses. tesis doctoral, Cádiz, Universidad de Cádiz.

BERBEIRA GARDÓN, J. L. (1996), Los verbos modales ingleses. Estudio semánticopragmático, Cádiz, Servicio de Publicaciones de la Universidad de Cádiz.

BERBEIRA GARDÓN, J. L. (1997), "Epistemic modality and discourse connectivity", Pragmalingüistica 3-4, 223-240.

BERBEIRA GARDÓN, J L. (1998a), "Relevance and modality", en Mateo Martínez, J. y F. Yus Ramos (eds.), Revista Alicantina de Estudios Ingleses 11, Special Issue Devoted to Relevance Theory, 3-22.

BERBEIRA GARDÓN, J. L. (1998b), Dimensiones pragmáticas de la gramaticalización, Lynx, Vol. 22, Valencia, Universidad de Valencia y Centro de Estudios sobre Comunicación Interlingüistica e Intercultural.

CARRETERO LAPEYRE, M. (1995), La pragmática de las expresiones de modalidad epistémica en el inglés hablado, tesis doctoral sin publicar, Madrid, Universidad Complutense.

EHRMAN, M. E. (1966), The Meanings of the Modals in Present-day American English, Janua Linguarum, Series Practica XLV, The Hague, Mouton.

GROEFSEMA, M. (1995), "Can, may, must and should: a Relevance-theoretic approach", Journal of Linguistics 31, 53-79.

HOYE, L. (1997), Adverbs and Modality in English, London and New York, Longman.

KLINGE, A. (1993), "The English modal auxiliaries: from lexical semantics to utterance interpretation", Journal of Linguistics 29, 315-357.

KRATZER, A. (1981), "The notional category of modality", in H.-J. Eikmejer and H. Rieser (eds.), Words, Worlds and Contexts, Berlin, Walter de Gruyter.

LEECH, G. N. (1971), Meaning and the English Verb, London, Longman.

PALMER, F. R. (1979) (1990), Modality and the English Modals, London and New York, Longman.

PAPAFRAGOU, A. (1997), "Modality in language development: a reconsideration of the evidence", UCL Working Papers in Linguistics 9, 77-105.

SPERBER, D. and D. WILSON (1986) (1995), Relevance. Communication and Cognition, Oxford, Blackwell.

SWEETSER, E. E. (1990). From Etymology to Pragmatics. Metaphorical and Cultural Aspects of Semantic Structure, Cambridge, Cambridge University Press.

WILSON, D. and D. SPERBER (1988), "Mood and the analysis of non-declarative sentences", in Dancy, J., Moravcsic, J. and C. Taylor (eds.), Human Agency: Language, Duty and Value, Stanford, Stanford University Press, 77-101.

Pragmalinguistica, 5-6, 1997-1998, 415-434 
WILSON, D. and D. SPERBER (1993), "Linguistic form and relevance", Lingua 90.1/2, 1-25.

Pragmalinguística, 5-6, 1997-1998, +15-434. 